that the best-fitting model was one in which cigarette smoking (or nicotine dependence) predicted depression. In the original study, we reported on analyses of nicotine dependence symptoms and symptoms of depression in order to maintain a focus on measures germane to psychiatry, in view of the scope of this Journal.

Finally, Dr Sheikh argues that depression must be caused by nicotine withdrawal rather than smoking. However, Benowitz ${ }^{3}$ has shown that active smokers go through several withdrawal phases during each day, and that these withdrawal phases are one of the factors that causes self-administration of nicotine. Therefore, it could also be argued that depressive symptomatology may be increased among active smokers because of this continual cycle of withdrawal and satiety.

1 Fergusson DM, Horwood LJ, Ridder EM. Tests of causal linkages between cannabis use and psychotic symptoms. Addiction 2005; 100: 354-66.

2 Fergusson DM, Boden JM, Horwood LJ. Structural models of the comorbidity of internalising disorders and substance use disorders in a longitudinal birth cohort. Soc Psychiatry Psychiatr Epidemiol in press.

3 Benowitz NL. Nicotine addiction. New Engl J Med 2010; 362: 2295-303.

Joseph M. Boden, David M. Fergusson, University of Otago, Christchurch,

New Zealand. Email: joseph.boden@otago.ac.nz

doi: 10.1192/bjp.198.1.73a

\section{Evolution and non-clinical psychotic symptoms}

In their recent editorial, Kelleher et al ${ }^{1}$ emphasised the importance of evolutionary theory for explaining the persistence of psychotic symptoms, depression and anxiety in humans. The authors did not mention the difference between proximate and ultimate explanations, in other words between 'how' and 'why' explanations, ${ }^{2}$ and this could make their argument for using evolutionary theory in psychiatric research more specific. In the development of treatments one needs an explanation at the proximate level, whereas the ultimate level can be necessary for generating hypotheses.

In evolutionary-based research the challenge is to find not which behaviour is beneficial now, but which behaviour has been advantageous for the procreation of ancestors in the past. This is the ultimate-level explanation. We know very little about our human ancestors and hypotheses can easily become 'just-so' stories with limited predictive value. Therefore rigorous testing at the how level is required. ${ }^{3}$ Furthermore, there are complicating factors such as cliff-edged fitness, ${ }^{4}$ whereby a limited number of traits is beneficial but too many are detrimental.

The possible theories for psychosis or schizophrenia mentioned by Kelleher et al vary enormously. It might have something to do with language development, complex social cognition, hypervigilance or with something completely different. However, all these theories need to be further developed to generate hypotheses at the how level, for example how language/hypervigilance/social cognition skills differ in humans with genes associated with schizophrenia or in family members of people with schizophrenia. The aim is to explain psychotic disorders at the proximate level, because that is needed to find the best possible treatment.

1 Kelleher I, Jenner JA, Cannon M. Psychotic symptoms in the general population - an evolutionary perspective. Br J Psychiatry 2010; 197: 167-9.

2 Mayr E. Cause and effect in biology. Science 1961; 134: 1501-6.

3 DeBruine L. Beyond 'just-so stories'. Psychologist 2009; 22: 930-3.

4 Nesse RM. Cliff-edged fitness functions and the persistence of schizophrenia. Behav Brain Sci 2004; 27: 862-3.

Dieneke Hubbeling, Consultant Psychiatrist, Wandsworth Crisis and Home Treatment Team, London, UK. Email: dieneke@doctors.org.uk

doi: 10.1192/bjp.198.1.74
Kelleher et al ${ }^{1}$ argue that evolutionary theories of psychosis provide a valuable theoretical framework for the investigation of non-clinical psychotic phenomena and that the findings of such research would generate new insights into the aetiology, nosology and treatment of psychosis. They rely mostly on Nesse's ideas of cliff-edged fitness ${ }^{2}$ and Dodgson \& Gordon's work on hypervigilance hallucinations ${ }^{3}$ and think that there must be an evolutionary advantage to non-clinical psychotic symptoms why would they otherwise be so prevalent in the population?

I would like to add two further possible explanations. The first is that non-clinical psychotic symptoms are neither advantageous nor disadvantageous and may have been passed on alongside other fitness enhancing phenotypes. The second is that non-clinical psychotic symptoms are disadvantageous but their negative effects are diminished by being coupled to advantageous phenotypes. Fodor \& Piattelli-Palmarini ${ }^{4}$ call this free-riding and argue that this is a counterexample to natural selection as proposed by Darwin (although Darwin was very well aware that non-adaptive processes play an important role in evolution).

Evidence to support one or the other evolutionary theory of mental illness will be hard to come by - I have argued elsewhere that, owing to its necessarily historical nature, it will be difficult to arrive at credible causal explanations. ${ }^{5}$

Even if evidence were available, there remains a considerable explanatory gap - why do some (young) people who experience non-clinical psychotic symptoms develop a full-blown psychotic illness with significant functional impairment? One of the aims of evolutionary psychiatry is to define mental disorder in valuefree terms. However, when assessing a patient's ability to function, values do come into play, as has been shown by Fulford. ${ }^{6}$ I think that whichever way one might argue the case, arriving at a psychiatric diagnosis is unlikely ever to make do without a notion of dysfunction.

As for the direct clinical utility of an evolutionary theory, I cannot see how knowing that psychotic symptoms might have been advantageous in times long gone is of huge benefit to patients or, for that matter, clinicians, and I think that current and possibly future treatment strategies work just as well without taking recourse to an evolutionary perspective.

I would like to thank Professor Ebmeier for his helpful suggestions and thoughts on evolutionary psychiatry.

1 Kelleher I, Jenner JA, Cannon M. Psychotic symptoms in the general population - an evolutionary perspective. Br J Psychiatry 2010; 197: 167-9.

2 Nesse RM. Evolution at 150: time for truly biological psychiatry. $\mathrm{Br} J$ Psychiatry 2009; 195: 471-2.

3 Dodgson G, Gordon S. Avoiding false negatives: are some auditory hallucinations an evolved design flaw? Behav Cogn Psychother 2009; 37 325-34.

4 Fodor JA, Piattelli-Palmarini M. What Darwin Got Wrong. Farrar, Strauss and Giroux, 2010

5 Treffurth Y. Evolution and psychiatry. Br J Psychiatry 2010; 196: 247

6 Fulford KWM. Moral Theory and Medical Practice. Cambridge University Press, 1989

Yvonne Treffurth, ST6 Child and Adolescent Psychiatry, Child and Adolescent Mental Health Learning Disability Team, The Phoenix Centre, Cambridge, UK. Email: yvonne.treffurth@gmail.com

doi: 10.1192/bjp.198.1.74a

I found the editorial by Kelleher et $a l^{1}$ both stimulating and thought provoking. However, it is important to bear in mind that a given characteristic must either promote or hinder an individual's chances of survival and procreation if it is going to have an impact on natural selection. Even if the presence of a 
certain trait - such as hypervigilance for possible dangers, as argued in the editorial - in a few members of a social group is potentially beneficial for the group, this would not affect the ability of each one of those hypervigilant individuals to spread their genes. At an individual level, it would be difficult to argue that hypervigilance would increase the overall chances of survival and procreation of a particular human. This individual would be cautious, but also seriously handicapped by an inability to trust others in the social group. Certain aspects of a human phenotype, such as psychotic symptoms, are not advantageous, but they have not been eradicated by evolution simply because they do not have a sufficient impact on survival before reproductive age. An evolutionary approach would not find any advantages in having bad teeth or weak coronary arteries, but the fact that these widespread human characteristics manifest themselves only after the individual has already had the chance to reproduce explains why it is that they are still - unfortunately - very much with us.

1 Kelleher I, Jenner JA, Cannon M. Psychotic symptoms in the general population - an evolutionary perspective. Br J Psychiatry 2010; 197: 167-9.

Rafael Euba, Consultant and Senior Lecturer in Old Age Psychiatry, Oxleas NHS Foundation Trust, Memorial Hospital, London, UK. Email: r.euba@ntlworld.com

doi: 10.1192/bjp.198.1.74b

Authors' reply: We thank readers for their interest in our editorial. ${ }^{1}$ The main purposes of the editorial were threefold: (i) to highlight the relatively recent identification and characterisation of a non-clinical psychosis population (for review see Kelleher \& Cannon $^{2}$ ); (ii) to point out that there might be important overlap in the genetics of the clinical and non-clinical psychosis phenotypes; and (iii) to discuss the potential value of this population for empirically testing evolutionary theories of psychosis.

Dr Euba points out that hypervigilance may lead to an individual being 'handicapped by an inability to trust others in the social group' and as a result being less likely to procreate. However, hypervigilance is not in itself a disadvantage. In fact, the more vigilant an animal, the more likely it is to identify threats such as predators and to protect both itself and its progeny, allowing the propagation of associated genes. Increasing levels of vigilance, however, would promote survival of the organism and its progeny only to a point. As this trait becomes ever more pronounced, it would eventually lead to the dysfunction identified by Euba - paranoia. Nesse referred to this as cliff-edged fitness, ${ }^{3}$ whereby traits may increase fitness up to a critical threshold, but beyond this point, fitness drops precipitously (the cliff edge here being the transition from hypervigilance into paranoia). Thus, while in its extreme form - paranoia - hypervigilance will be negatively selected owing to negative fitness consequences, a 'subthreshold' level of this trait would be positively selected.

We agree with Treffurth that it is possible that non-clinical psychotic symptoms may be neither advantageous nor disadvantageous and that associated genes may have been passed on alongside other fitness-enhancing phenotypes. Our argument, however, is that if, as has been suggested by many researchers to date, the genetics of psychosis encode for positive as well as negative traits, then people with the recently characterised nonclinical psychosis phenotype may provide a valuable population in which to conduct empirical research.

Hubbeling makes the very point that we wished to emphasise in our editorial - that the non-clinical psychosis phenotype provides us with a population in which to test hypotheses about the evolutionary benefit of psychosis genes. It is clear why genes that promote certain traits, such as language development, hypervigilance and complex social cognition, would be selected in evolution. The 'how' questions, as Hubbeling points out, require attention, for instance how these traits differ in (non-psychotic) persons with psychosis genes compared with persons without (or with fewer) psychosis genes. This type of research is precisely what we wish to encourage by highlighting the validity of the nonclinical psychosis phenotype for empirical investigation. This population provides a potentially valuable means for moving beyond 'just-so' stories ${ }^{4}$ into the realm of testable hypotheses.

1 Kelleher I, Jenner JA, Cannon M. Psychotic symptoms in the general population - an evolutionary perspective. Br J Psychiatry 2010; 197: 167-9.

2 Kelleher I, Cannon M. Psychotic-like experiences in the general population: characterizing a high-risk group for psychosis. Psychol Med 2010; May 19: 1-6 (Epub ahead of print).

4 Kipling R. Just So Stories. Macmillan, 1902.

3 Nesse RM. Cliff-edged fitness functions and the persistence of schizophrenia. Behav Brain Sci 2004; 27: 862-3.

Mary Cannon, Associate Professor of Psychiatry and Consultant Psychiatrist, Royal College of Surgeons in Ireland and Beaumont Hospital, Dublin, Ireland. Email: marycannon@rcsi.ie; Ian Kelleher, Department of Psychiatry, Royal College of Surgeons in Ireland, Dublin, Ireland; Jack A. Jenner, Department of Psychiatry, University Medical Center Groningen and the Mental Health Care Foundation in Friesland, The Netherlands.

doi: 10.1192/bjp.198.1.75

\section{Corrections}

Auditory hallucinations and brain structure in schizophrenia: voxelbased morphometric study. BJP, 196, 412-413. All correlations reported in this paper are negative (i.e. the higher the hallucination scores, the smaller the gray matter values). There were no positive correlations.

Nalmefene in the treatment of pathological gambling: multicentre, double-blind, placebo-controlled study. BJP, 197, 330-331. Page 330 , col. 1, the fifth sentence should read: 'In a double-blind, 16-week multicentre trial $(n=207)$, various doses of nalmefene $(25,50,100 \mathrm{mg} /$ day $)$ showed similar efficacy, but premature discontinuation was common (drop-out rate: $66 \%) .{ }^{4}$, 